The fields of vision show slight concentric contraction. In each eye there is a large central scotoma, absolute for red and green, partial for blue, and relative for white. The nasal sinuses have been examined by Mr. F. Sydenham who reports that there is nothing abnormal. Mr. Roper Hall extracted eight septic teeth, but there has been no improvement from this treatment.

In this case, from a medico-legal point of view, it is impossible to exclude the septic condition of the arm as a cause of the defective vision, and the fact that the sight began to fail during the period of septic absorption from the arm, strongly suggests a causal connection. It is of course possible that the condition of the teeth may have had something to do with the sequence of events, but I cannot get away from the fact that the septic arm and the loss of sight coincided in point of time. My report was that in my opinion the blindness was directly caused by the accident.

Quite recently I was asked to see a case of acute glaucoma in a woman who was recovering from an operation for appendicitis. Her wound was suppurating and the green pus contained bacillus pyocyaneus. As there was no perception of light I concluded that there was something more than glaucoma of but 36 hours' duration. The eye yielded to eserin treatment and in two days I could see the fundus and discovered an optic neuritis. A week later the patient died suddenly from embolism. Here we have a case of a septic wound causing an optic neuritis perhaps of embolic nature, and if an abdominal wound can have this effect it is reasonable to suppose than a septic arm may act in similar fashion. In my case the retrobulbar neuritis was associated with a neuritis of the nerve head, an unusual combination.

\title{
EXCISION OF THE SUPERIOR TARSUS AND CONJUNCTIVA IN THE TREATMENT OF TRACHOMA
}

BY

\section{G. Freeman Heal, M.D., C.M., D.O.M.S.}

WhILE in Bagdad in $1924 \mathrm{I}$ was for some months in charge of the Ophthalmic Department of the Iraq Government Hospital where large numbers of Arabs attend for treatment, almost all of whom have trachoma. The majority of the natives of the country live their whole lives amid surroundings which are too insanitary and squalid for their condition to be comprehensible to a western mind. Apart from the ineffective ritualistic washing imposed upon them by their religion, Arab men and women never cleanse either 
their bodies, their clothes, or their children. Their children instead of being washed have their faces tattooed, and their eyes smeared with greasy pigments to beautify them. This lack of cleanliness is undoubtedly one of the strongest aetiological factors in the spread of trachoma. It is a great rarity to find any native, at least in Bagdad, who is free from trachoma. Those who attend hospital generally have the disease in an aggravated form with one or more complications, among which trichiasis is probably the most distressing. The urgent necessity of relieving if possible some of this distress in a more effective way than by the routine use of the copper sulphate stick was very much impressed upon me, and I determined to try the effect of removal of tarsus and conjunctiva. This is a method of treatment originated by Heisrath and advocated by MacCallan. The difficulty I encountered was to find any description of a simple and satisfactory method of performing the operation or any details of its special difficulties or complications. Ultimately I succeeded in elaborating a satisfactory method of performing the operation which I did in fifty cases. All of these cases, with one exception gave the most gratifying results. I was unable to follow the cases for more than a month or so because I left the country, but the observed benefits were so manifest, and the likelihood of undesirable after-effects so remote that I venture to describe the results and to recommend the technique of the procedure as applicable both to the heavily infected East, and to many of the sporadic cases which occur at home. (One case done recently in England for severe trichiasis was entirely satisfactory.)

\section{Indications}

The operation is particularly indicated in active trachoma with a thickened or deformed tarsus. It is by far the most satisfactory way of treating trichiasis and I should operate on any case, quiescent or active, in which this complication was present.

I have no experience of the operation in very early cases of the disease and would not recommend it in such cases until the ordinary method of treatment had been given a trial.

There is a form of trachoma, seen only occasionally, in which there are no visible follicles, the palpebral conjunctiva is thin, bluish and angry, the tarsal plate is not perceptibly thickened, there is slight if any trichiasis, vascular pannus is present without any trachomatous infiltration of the cornea, irritation and photophobia are marked. Almost any active treatment seems to disagree with this form of trachoma, and I should hesitate to operate on it unless trichiasis were present. Otherwise than in this somewhat rare type of case the results are uniformly good. Subjective symptoms are relieved, pannus is much improved, a 
hazy steamy cornea will often clear considerably, trichiasis is entirely cured, and the eye becomes quiet. In the East the Arabs are so gratified that they will fetch their relations to be operated on.

\section{Operation}

Anaesthetic.-Local infiltration with 2 per cent. novocain and adrenalin. Care must be taken to ensure infiltration of the lateral extremities of the tarsus as these sometimes escape the anaesthetic and give pain when cut. The conjunctival sac is anaesthetized with cocain in the usual way.

Preparation of Patient.-The skin of the face and lids should be thoroughly cleansed and painted with iodine. A towel with aperture for access to eye should cover face and hair.

Instruments.-Hypodermic syringe with long fine sharp needle for infiltration of lid with novocain. MacCallan's entropion spatula. 'Two pairs toothed conjunctival fixation forceps. Beer's cataract knife. A stout pair of toothed dissecting forceps for gripping the tarsal plate. Conjunctival scissors, fine pointed, not sharp pointed. Needle holder. Three curved needles. Ophthalmic silk No. 2.

Operation.-The upper lid is everted and raised on the lid spatula passed beneath it as high as the fornix. The spatula stretching the lid helps to prevent haemorrhage as well as affording protection to the globe.

Holding the knife vertically make an incision through the conjunctiva and tarsal plate extending the whole length of the lid margin and placed $1.5 \mathrm{~mm}$. above the openings of the meibomian glands. This leaves a rim of tarsus in the lid margin for support. Insert one limb of the strong dissecting forceps into the centre of the incision and grip the tarsal plate. The operator now controls the tarsal plate with the left hand and holds the scissors in his right. The assistant with a pair of conjunctival forceps in his left hand holds backward the lid margin and its lashes. His right hand controls the lid spatula. With the points of the scissors pressed firmly against the tarsus, clear, by snipping from below upwards, all the attachments of the levator palpebrae and the tissues of the lid from the back of the tarsal plate. This dissection is continued above the tarsal plate so as to raise the conjunctival membrane almost as high as the fornix. Care must be exercised at this stage to avoid button-holing the conjunctiva. Next sever the lateral insertions of the tarsal plate with one snip of the scissor points at each extremity. Three sutures are next inserted from the skin surface of the lid, a central and two lateral. The central stitch is inserted first. Pass the armed curved needle through the skin and tissues of the lid about $5 \mathrm{~mm}$. above the lid margin and just lateral to its centre, pick up the free conjunctiva 


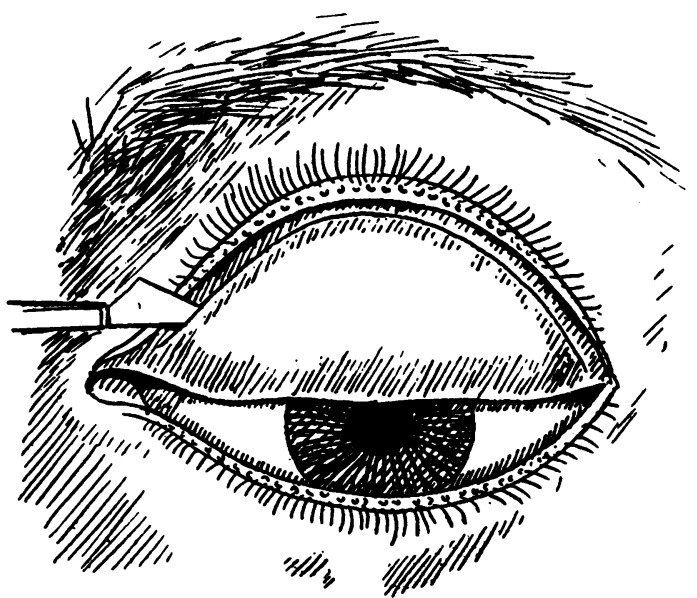

Fio. 1

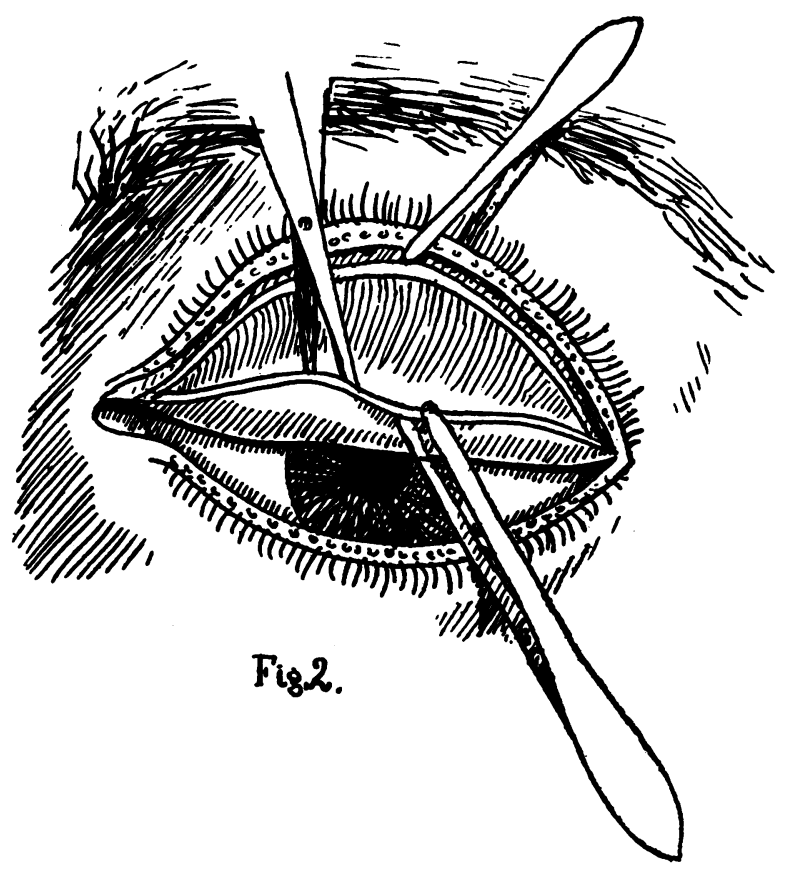


$3 \mathrm{~mm}$. to $5 \mathrm{~mm}$. above the tarsal plate and return the needle through the lid to emerge a few $\mathrm{mm}$. medial to its point of entry. The lateral sutures are inserted in a similar manner, but these

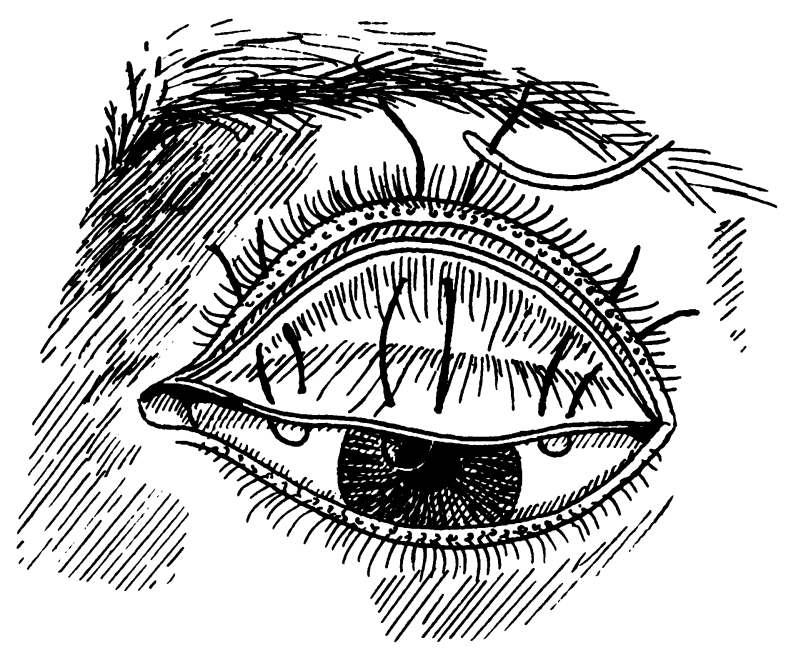

Fig.3.

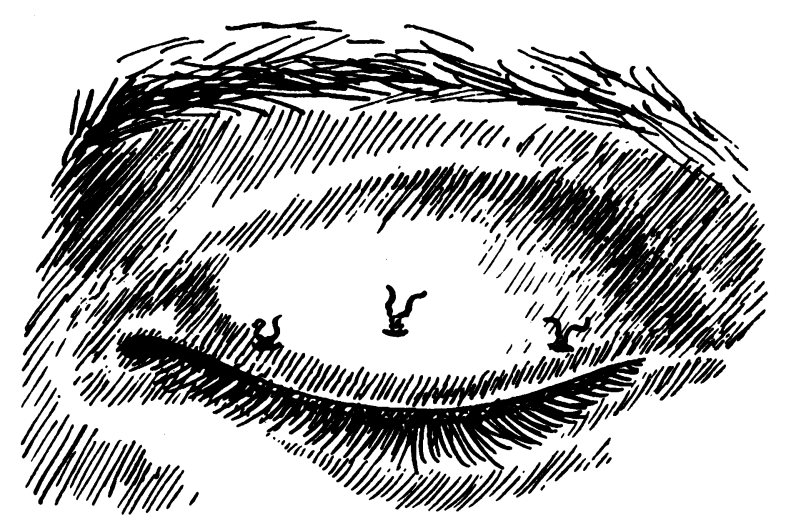

Fig. 4.

enter and emerge from the skin at a level nearer the lid margin $(2-3 \mathrm{~mm}$. from it)). The tarsus is now removed by cutting through the conjunctiva above its upper border, taking care to avoid the stitches. Next the three sutures are firmly tied; they should have been so inserted and tied that they cause the free margin of the lid to be rolled outward, thus correcting any trichiasis 
present. This is one of the important points in inserting the sutures and the accomplishment of this eversion has to be constantly kept in mind during their insertion. A pad of gauze smeared with sterile vaseline is now bandaged over the eye.

\section{After Treatment}

The eye is dressed and bathed daily.

Hot fomentations are used if there is much swelling. The stitches are removed on the fourth day, the dressings on the fifth.

\section{Remarks}

Lids which have been subjected to previous operations offer considerable difficulty. They usually show loss of tissue and cicatrization which makes a further removal of tissue impossible if the lids are to remain capable of closing over the globe after the operation. Even in some of these cases, however, the removal of a small distorted tarsus and not too much conjunctiva will cure the trichiasis and leave lids which act as efficient coverings for the globe if the stitches be inserted somewhat higher so as to draw down the lid tissues in their grasp.

After the above operation where an area is left on the inner surface of the lid which is devoid of an epithelial covering one might expect to get cicatrization and contraction which would eventuate in a secondary entropion. This does not occur. The thickened puckered tarsus buried in the supple tissues of the lid, which themselves have undergone no reduction or contraction but rather the reverse, acts like a vicious framework, rolling in the lid and maintaining the entropion. The slight cicatrization following the removal of this framework is so small in comparison with the cicatrization and deformity of the original tarsus that the gain is sufficient to maintain the cure of the trichiasis.

The reasons for the arrest of the trachomatous process and the marked improvement following the operation are not altogether clear. Where the tarsus is hypertrophied to a mass of ischaemic and partially degenerated scar tissue it seems reasonable to suppose that it acts as a foreign body in the lid. On its removal the cornea is covered by a soft fleshy curtain and not by a hard resisting visor, hence the improvement in the pannus. The operation itself causes a local blood letting and brings an increased supply to the part. Such suggestions, however, are hardly a sufficient explanation.

After the operation the fornix is drawn forward and the lid is not so easily everted as previously. Eversion can still be performed however with the aid of a glass rod. There is no interference 
with the movements of the globe by reason of the advancement of the fornix.

There is no danger of ptosis following the operation as above described. On the contrary the trachomatous ptosis so often present is relieved, the lid is raised and the palpebral aperture widened. There is a tendency unless the central stitch be put in rather higher than the others for the centre of the lid margin to be drawn up in a shallow concave notch. This is undesirable from a cosmetic point of view.

The operation wound heals rapidly and the raw area quickly becomes covered with epithelium which can grow inwards from its whole circumference.

Bandages themselves tend by their pressure to invert the lids and they are therefore discarded as early as possible.

Occasionally a little knob of granulation tissue springs up on the under surface of the lid and has to be snipped off.

\title{
CROSSED QUADRANT HEMIANOPSIA
}

\author{
BY \\ DR. C. H. FELIX \\ THE HAGUE, HOLLAND
}

OF this eye disease there are only two cases known in literature. I am in a position to add a third.

Mr. V. K., aged 63 years, is suffering from arteriosclerosis and degeneration of the heart. After reading on the evening of April 13, 1924, he retired to bed and awakened the next morning totally blind. The patient was very calm, abnormally calm under these circumstances, whilst his wife was very nervous and wanted to call the doctor immediately. The patient thought that this was not necessary. Noticing that his calmness was very strange under the situation, he answered: "Oh, I have gone through so much that this can also come."

To this very apathetic turn of mind I shall refer later. At about ten o'clock in the morning, when the family doctor visited him, the patient began to see a little again. He could not distinguish the figure of the doctor, but could observe the glistening of his eyeglasses. Gradually the situation became better and after a few days he was able to see large objects and persons as in a mist.

Bronchitis with a disorderly action of the heart kept him in bed for a long time. Not before August 2, 1924, was I able to examine 